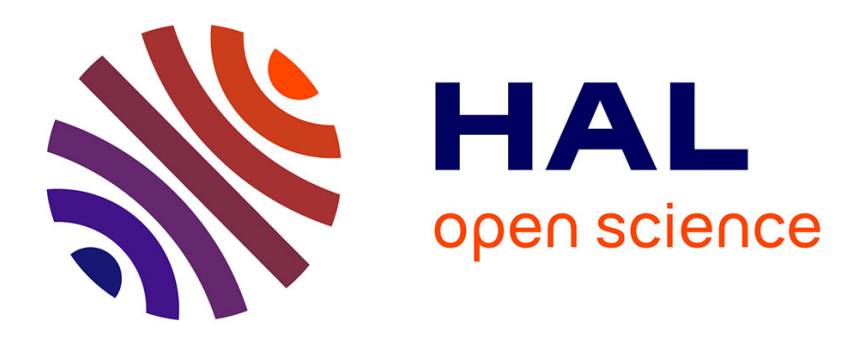

\title{
Multidrug-resistant Gram-negative bacteria: How to treat and for how long \\ Helen Giamarellou
}

\section{To cite this version:}

Helen Giamarellou. Multidrug-resistant Gram-negative bacteria: How to treat and for how long. International Journal of Antimicrobial Agents, 2010, 36, 10.1016/j.ijantimicag.2010.11.014 . hal00650375

\section{HAL Id: hal-00650375 https://hal.science/hal-00650375}

Submitted on 10 Dec 2011

HAL is a multi-disciplinary open access archive for the deposit and dissemination of scientific research documents, whether they are published or not. The documents may come from teaching and research institutions in France or abroad, or from public or private research centers.
L'archive ouverte pluridisciplinaire HAL, est destinée au dépôt et à la diffusion de documents scientifiques de niveau recherche, publiés ou non, émanant des établissements d'enseignement et de recherche français ou étrangers, des laboratoires publics ou privés. 


\section{Accepted Manuscript}

Title: Multidrug-resistant Gram-negative bacteria: How to treat and for how long

Author: Helen Giamarellou

PII: S0924-8579(10)00475-9

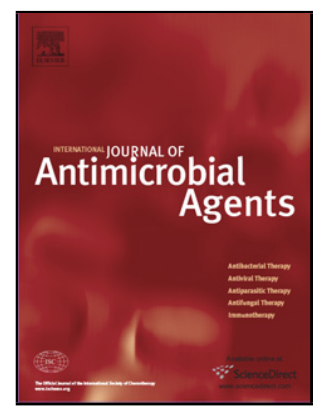

DOI: doi:10.1016/j.ijantimicag.2010.11.014

Reference: ANTAGE 3468

To appear in:

International

Journal

of

Antimicrobial

Agents

Please cite this article as: Giamarellou H, Multidrug-resistant Gram-negative bacteria: How to treat and for how long, International Journal of Antimicrobial Agents (2010), doi:10.1016/j.ijantimicag.2010.11.014

This is a PDF file of an unedited manuscript that has been accepted for publication. As a service to our customers we are providing this early version of the manuscript. The manuscript will undergo copyediting, typesetting, and review of the resulting proof before it is published in its final form. Please note that during the production process errors may be discovered which could affect the content, and all legal disclaimers that apply to the journal pertain. 
Multidrug-resistant Gram-negative bacteria: How to treat and for how long

Helen Giamarellou*

Athens University Medical School, Athens, Greece

* Tel.: +30 2106867946 .

E-mail address: e.giamarellou@hygeia.gr. 


\section{Abstract}

The emergence of multidrug-resistant (MDR) Gram-negative bacilli creates a big problem for the treatment of nosocomial infections. As the pharmaceutical pipeline wanes, the only therapeutic options are two revived antibacterials (colistin and fosfomycin), a newer one (tigecycline) and an early-phase neoglycoside (ACHN-490). Polymyxins, known since 1947, are mostly represented by polymyxin $E$ (colistin), which has recently gained a principal position in the management of the most difficult-to-treat MDR Gram-negative pathogens - Pseudomonas aeruginosa, Acinetobacter baumannii and Klebsiella pneumoniae. However, despite promising therapeutic results in $59-75 \%$ of cases, the reported studies share common drawbacks, i.e. the absence of a control group, their retrospective nature, variable dosing and duration of therapy, simultaneous administration of other antibiotics in $>70 \%$ and a lack of resistance development monitoring. The necessity for well-designed prospective clinical trials is therefore urgent. Fosfomycin is active in vitro against MDR Enterobacteriaceae, including a high proportion of $P$. aeruginosa; however, clinical experience is lacking with the parenteral formulation in MDR infection and on the best combinations to prevent resistance development. Tigecycline, which is active against MDR Enterobacteriaceae and $A$. baumannii, has shown satisfactory clinical experience. However, dosage adjustment is required because of low blood levels. ACHN-490, which has promising in vitro activity against MDR K. pneumoniae, is still in early phase II trials in urinary tract infections. Meanwhile, the strict application of infection control measures is the 
cornerstone of nosocomial infection prevention, and antibiotic stewardship, exemplified by appropriate duration of therapy and de-escalation policies, should not be overlooked.

Keywords:

Multidrug-resistant bacteria

Colistin

Tigecycline

Fosfomycin

Carbapenems

Procalcitonin 


\section{Introduction}

In 2010, infections caused by multidrug-resistant (MDR) bacteria continue to challenge physicians and endanger their patients' lives [1]. During the last decade efforts to combat microorganisms focused mainly on Gram-positive bacteria, and drug companies developed several novel antimicrobial agents to fight them. Unfortunately, the growing problem of multidrug resistance in Gramnegative bacteria was not paralleled by the development of novel antimicrobials. The return to the pre-antibiotic era has become a reality in many parts of the world. MDR microorganisms were recently named as the 'ESKAPE' pathogens (Enterococcus faecium, Staphylococcus aureus, Klebsiella pneumoniae, Acinetobacter baumannii, Pseudomonas aeruginosa and Enterobacter spp.), indicating their 'escape' from the effects of antibacterial agents or the nonexistence of newer active antibiotics [2].

Data published in 2004 by the US National Nosocomial Infection Surveillance System report resistance rates among $P$. aeruginosa isolates to imipenem and quinolones as $21.1 \%$ and $29.5 \%$, respectively. In intensive care unit (ICU) isolates the respective rates of resistance were even higher (up to $51.6 \%$ for ciprofloxacin, $31.4 \%$ for piperacillin/tazobactam, $38 \%$ for imipenem and $23.6 \%$ for ceftazidime) [3]. Relevant figures for ICU isolates of $P$. aeruginosa derived from Europe are even worse, as from 1990 to 1999 resistance to aminoglycosides reached $37-70 \%$, ceftazidime $57 \%$, piperacillin/tazobactam $53 \%$, ciprofloxacin $56 \%$ and imipenem 52\% [4]. 
Multicentre surveillance studies have reported the proportion of imipenemresistant $A$. baumannii strains to be as high as $85 \%$ in bloodstream isolates from ICU patients in Greece, and $48 \%$ in clinical isolates from hospitalized patients in Spain and Turkey [5]. Among 33 European countries participating in the European Antimicrobial Resistance Surveillance System (EARSS) in 2007, six reported carbapenem resistance rates of $>25 \%$ among $P$. aeruginosa isolates, the highest being reported from Greece (51\%). According to EARSS, Greece also has the highest resistance rates among $K$. pneumoniae: $46 \%$ to carbapenems, $58 \%$ to quinolones and $63 \%$ to third-generation cephalosporins [5].

Based on our very weak antimicrobial armamentarium, this review is mainly focused on three compounds: colistin, a re-emerging old antibacterial; tigecycline, a genuinely new antibacterial; and fosfomycin, an old antibacterial being revived.

\section{Colistin}

The emergence of MDR Gram-negative bacilli has led to the revival of the polymyxins, an old class of cyclic polypeptide antibiotics discovered in 1947 . The group consists of polymyxins $A-E$, of which only polymyxin $B$ and polymyxin $E$ (colistin) are currently on the market [6]. Colistin is available in two forms: colistin sulfate (tablets or syrup for bowel decontamination and powder for topical use) and colistin methanesulfonate (International Nonproprietary Name: colistimethate 
sodium) for parenteral use. In the USA and Brazil, polymyxin B sulfate is also used parenterally [7].

Colistin is active in vitro against Enterobacteriaceae (including carbapenemaseproducing strains), Haemophilus influenzae, Legionella pneumophila, MDR P. aeruginosa and Acinetobacter spp., and Stenotrophomonas maltophilia, including most of the pan-drug-resistant (PDR) strains $[8,9]$.

The in vitro interaction of colistimethate sodium with rifampicin has been evaluated against PDR $P$. aeruginosa strains (including colistin). Synergy depending on exposure time was reported in $11.8-41.7 \%$ of strains [9]. Synergy has also been reported between colistin and 1) rifampicin in MDR A. baumannii strains producing OXA-58 carbapenemase, 2) minocycline in imipenem-resistant A. baumannii clinical isolates and 3) meropenem against $P$. aeruginosa and $A$. baumannii strains $[10,11,12]$.

From 1999 until August 2005, in seven retrospective studies involving almost 300 patients without cystic fibrosis, of whom most were ICU patients with ventilatorassociated pneumonia, intravenous colistimethate sodium was given at a dose of 1-3 million IU every $8 \mathrm{~h}$ for $12-22$ days $[1,13]$. Either MDR $P$. aeruginosa or MDR A. baumannii were isolated in almost all patients at a rate close to $50 \%$. Clinical cure rates ranged between 57 and $73 \%$, with mortality rates of $20-62 \%$. Clinical efficacy exceeding $50 \%$ in nosocomial pneumonia was comparable with previously reported rates with piperacillin/tazobactam, imipenem/cilastatin and ciprofloxacin. However, it should be pointed out that almost all the reported 
studies share the same drawbacks: 1) absence of a control group, 2) retrospective design, 3) variable dosing and duration of therapy, 4) simultaneous administration of other antibiotics (mostly imipenem) in $70-100 \%$ of cases, 5 ) no monitoring of resistance development during and at the end of therapy and 6) a wide range of nephrotoxicity, which owing to the retrospective character of the studies cannot be attributed exclusively to colistin.

In 2007, two retrospective monotherapy studies with colistimethate sodium were published. In the first, no difference in mortality rates ( $51.6 \%$ vs $45.1 \%)$ was observed between two groups: 31 patients with ventilator-associated pneumonia caused by isolates susceptible only to colistin, who were treated with colistimethate sodium monotherapy, and 30 patients with ventilator-associated pneumonia caused by carbapenem-susceptible strains, who were treated with imipenem/cilastatin or meropenem as monotherapy [14]. In the second study the efficacy of monotherapy with colistimethate sodium was compared with imipenem in ventilator-associated pneumonia caused by isolates susceptible only to colistin $(N=60)$ or carbapenem-susceptible $(N=60) A$. baumannii $(51.6 \%$ vs $61.7 \%$ ) or $P$. aeruginosa (48.4\% vs $38.3 \%$ ). A favourable clinical response was observed in $75 \%$ versus $71.7 \%$ of patients in the two study groups [15].

Colistimethate sodium was also studied retrospectively in 95 cancer patients with MDR $P$. aeruginosa [16]. Patients were treated with either colistin $(N=31,45 \%$ neutropenic) or at least one active antipseudomonal agent (control group, $N=64$, $37 \%$ neutropenic). Patients treated with colistin monotherapy had higher clinical and microbiological responses than those in the control group (52\% vs $31 \%$ and 
$48 \%$ vs $41 \%$, respectively). However, none of the differences in end points reached statistical significance. Multiple logistic regression analysis showed that patients treated with colistin were 2.9-fold more likely than patients in the control group to experience a clinical response to therapy $(P=0.026)$. A major limitation of the study, however, was the lack of evaluation of the time to initiate adequate therapy.

In the effort to address the issue of colistimethate sodium monotherapy vs combination therapy, a recent meta-analysis revealed no statistical difference in cure rates when colistimethate sodium alone was compared with the combinations with meropenem, piperacillin/tazobactam or ampicillin/sulbactam [17].

The efficacy of aerosolized colistin in the non-cystic fibrosis patient is currently a matter of concern, since solid data from prospective studies are lacking. However, aerosolized colistin (given with a specific vibrating nebulizer) should be thought of as adjunctive to intravenous therapy in patients with ventilatorassociated pneumonia due to MDR Gram-negative bacteria that are susceptible to colistin [18].

The results of a literature review of the treatment of MDR A. baumannii central nervous system (CNS) infections in 32 patients are of great importance. Colistimethate sodium was given at a dose of $2.5-5.0 \mathrm{mg} / \mathrm{kg}$ per day intravenously or 3.5-10 mg intrathecally every $12-24 \mathrm{~h}$ and $5-20 \mathrm{mg} / \mathrm{day}$ 
intraventricularly for 15-63 days. Thirty of the 32 patients were cured, with CNS sterilization, in 1-6 days (median 4.1 days) [19].

It should be mentioned that colistimethate sodium $2.4 \mathrm{mg}$ contains $1 \mathrm{mg}$ colistin base, and that pure colistin base has a potency of $30000 \mathrm{IU} / \mathrm{mg}$ and colistimethate sodium $12500 \mathrm{IU} / \mathrm{mg}[1,13]$. Because of the lack of accurate pharmacokinetic and pharmacodynamic information in non-cystic fibrosis patients, the optimal dosage of colistin is unclear. Colistimethate sodium in patients with normal renal function is usually given in the USA at a dose of 2.5$5.0 \mathrm{mg} / \mathrm{kg}$ per day intravenously in two to four equal doses [1,13]. The Greek experience has proved that a higher dose of 3 million IU every $8 \mathrm{~h}$ can be safely administered [13].

In a recent study in 18 critically ill ICU patients, where colistin concentrations were measured in plasma by a novel rapid chromatography-tandem mass spectrometry method, the half-life of colistimethate sodium disposition was $2.3 \mathrm{~h}$ and for colistin was $14.4 \mathrm{~h}$. The predicted $\mathrm{C}_{\max }$ was $0.60 \mathrm{mg} / \mathrm{L}$ after the first dose of 3 million IU colistimethate sodium and $2.3 \mathrm{mg} / \mathrm{L}$ following repeated administration of 3 million IU every $8 \mathrm{~h}$. The results indicate that colistin concentrations generally remain below the MIC breakpoint $(2 \mathrm{mg} / \mathrm{L})$ after the first few doses of the currently used dosing regimen [20]. These observations are a matter of concern, particularly for critically ill patients in whom adequate antimicrobial therapy within the first hour of septic shock is required. Therefore a loading dose of 9 million IU and a maintenance dose of 4.5 million IU every $12 \mathrm{~h}$ 
has been suggested, resulting in the same average steady-state concentration of colistin as the current dosing schedule, but achieved more quickly [20].

The intrathecal and intraventricular doses of colistimethate sodium are equal to $125000-250.000 \mathrm{IU} /$ day, whereas by the inhalation route the recommended dosage ranges from $500000 \mathrm{IU}$ every $12 \mathrm{~h}$ to 2 million IU every $8 \mathrm{~h}$ [19]. Colistin kinetics in the various body compartments remains a poorly investigated field, requiring prompt exploration $[1,3]$.

Recent data indicate that nephrotoxicity in ICU patients after colistimethate sodium administration ranges from 0 to $36 \%[1,13]$, while in a recent review focusing on polymyxin nephrotoxicity, an even wider range was reported (15$58 \%)$ [21]. The incidence of neurotoxicity in earlier studies of colistin reached approximately $7 \%$. Both nephro- and neurotoxicity seem to be dose-dependent and reversible $[1,3]$.

As early as 2007 the excessive use of colistin (owing to the frequent isolation of MDR pathogens in a Greek ICU) led to the emergence of colonization with colistin-resistant K. pneumoniae in the bronchial and bowel floras of $37 \%$ of patients [22]. The occurrence of various infections with colistin-resistant Gramnegative bacteria, and breakthrough bacteraemia with Proteus and Serratia spp. intrinsically resistant to colistin in patients receiving treatment with colistin for $>12$ days (median duration of therapy 27 days), was certainly worrying [23].

Also of concern is the emergence in Greek ICUs since 2001 of K. pneumoniae strains producing metallo- $\beta$-lactamases (MBLs) and, more recently, $K$. 
pneumoniae carbapenemases (KPC), which render K. pneumoniae strains resistant to all antibiotics except colistin $[24,25]$. Horizontal transmission of PDR Klebsiella through caregivers' hands was also proved [25]. The analysis of risk factors after a Greek ICU outbreak of PDR $P$. aeruginosa causing ventilatorassociated pneumonia revealed that the sole independent predictors were the administration of colistin for $\geq 13$ days or the combined use of colistin with a carbapenem for $>20$ days [26]. Additionally, in a recent matched case-control study, the use of colistin for $>14$ days was identified as the only independent risk factor in the multivariable model $(P=0.002)$ [27]. For the survival of colistin in the hospital, and particularly the ICU, colistin should be given only in case of isolation of MDR strains sensitive only to colistin, or empirically in nosocomial or ICU late septic shock (particularly in late ventilator-associated pneumonia whenever risk factors for MDR Gram-negative organisms are present, such as preceding ventilator-associated pneumonia episodes or preceding therapy with a carbapenem for $>10$ days).

\section{Tigecycline}

Tigecycline is a parenteral minocycline analogue that holds promise as monotherapy for patients with serious polymicrobial infections, including MDR microorganisms. Its Gram-negative spectrum includes MDR A. baumannii, extended-spectrum $\beta$-lactamase (ESBL)-producing Enterobacteriaceae, and KPC- and VIM-producing K. pneumoniae and S. maltophilia strains $[28,29]$. However, an increase in tigecycline resistance among Enterobacter and Klebsiella spp. was documented from 2001 to 2006 in many parts of the world. 
Data from TEST 2005-2007 (Tigecycline Evaluation and Surveillance Trial) indicate that the tigecycline $\mathrm{MIC}_{90}$ for $A$. baumannii remained stable $(\leq 2 \mathrm{mg} / \mathrm{L})$ [30]. Depending on the resistance cutoff ( $2 \mathrm{mg} / \mathrm{L}$ or $4 \mathrm{mg} / \mathrm{L}$ ), $80.9 \%$ or $93.1 \%$ of A. baumannii strains, respectively, are considered to be susceptible [31]. On the other hand, tigecycline was $100 \%$ active against a total of 104 carbapenemaseproducing (serine $\beta$-lactamase and $\mathrm{MBL}$ ) strains of Enterobacteriaceae collected from 2000 to 2005 [32].

Tigecycline is available only as an intravenous formulation and is administered as a $50 \mathrm{mg}$ 1-hour infusion every $12 \mathrm{~h}$ after an initial loading dose of $100 \mathrm{mg}$. After a $50 \mathrm{mg}$ dose tigecycline exhibits linear pharmacokinetics, with $\mathrm{C}_{\max }$ at $0.62 \pm 0.09 \mathrm{mg} / \mathrm{mL}$ and a half-life of $37 \pm 12 \mathrm{~h}$. Kinetics of tigecycline in the bile, gall bladder and colon are promising but bone and lung concentrations are still controversial $[33,34]$.

From January 2007 to April 2007 nine studies related to MDR Gram-negative infections were published or available online [1]. However, most of them are retrospective and non-comparative, with little use of monotherapy, rendering the true role of tigecycline in the outcomes very obscure. Additionally, the MICs of tigecycline for the targeted pathogens were not universally available, leading to different definitions of multidrug resistance across the studies. In order to bypass some of these problems a retrospective study in three Greek tertiary hospitals was performed [35]. Among patients treated with tigecycline, 45 adults (35 in ICU) met strictly defined criteria for infection with MDR Gram-negative pathogens. They received tigecycline at the standard dose for $28 \mathrm{~A}$. baumannii 
and $23 \mathrm{~K}$. pneumoniae infections ( $>1$ pathogen isolated in 6 cases) with an MDR or PDR profile (21 ventilator- and healthcare-associated pneumonia, 10 bloodstream infections and 14 surgical infections). Tigecycline MIC values among isolates of $A$. baumannii ranged from 1 to $8 \mathrm{mg} / \mathrm{L}$, whereas those for $K$. pneumoniae ranged from 0.5 to $3 \mathrm{mg} / \mathrm{L}$. Successful clinical response rates of $90.5 \%$ and $80 \%$ were recorded for ventilator-/healthcare-associated pneumonia and bloodstream infection, respectively, with an overall successful clinical response of $80 \%$. Cumulative successful microbiological outcomes were lower than clinical success rates because there were 13 episodes of superinfection and breakthrough infection among 10 patients.

Despite tigecycline's promising clinical efficacy, the possibility of gastrointestinal adverse events, i.e. nausea, vomiting, diarrhoea and anorexia [36], as well as decreased fibrinogen levels (Giamarellou $\mathrm{H}_{\text {., }}$ unpublished data), should be of concern. The low tigecycline levels attained in the serum are probably the driving force for the development of resistance while on treatment, particularly whenever the MIC for the targeted pathogen exceeds the $\mathrm{C}_{\max }$ of the drug, which is often observed in A. baumannii strains [37].

Clinicians should adopt a cautious approach in off-label use of tigecycline owing to the currently limited evidence of efficacy. Interestingly, in the Greek cohort of 45 patients treated with tigecycline for MDR or PDR infections, 10 episodes of superinfection with pathogens inherently resistant to tigecycline were observed (i.e. Proteus spp., Providencia spp., P. aeruginosa etc.) [35]. This suggests 1) use of the drug as monotherapy be restricted to patients with documented non- 
pseudomonal infections, and 2) the addition of an antipseudomonal agent to empirical regimens in patients with risk factors for pseudomonal infections. Especially in settings with MDR epidemiology (particularly in institutions with KPC predominance), an aminoglycoside or colistin appear to be the most attractive combination for tigecycline.

\section{4) Other antimicrobial agents}

The answer to the clinician's question of whether carbapenems can be used against VIM-producing KPC (VPKP) MDR Klebsiella pneumoniae isolates was investigated in a recent prospective observational study [38,39]. A total of 162 consecutive patients with bloodstream infections were identified. Sixty-seven (41.4\%) were infected with VPKP and 95 (58.6\%) with non-VPKP. Fourteen were infected with carbapenem-resistant (imipenem or meropenem) VPKP (MIC $>4 \mu \mathrm{g} / \mathrm{ml}$ ). Among the 67 patients infected with VPKP-positive isolates, 49 (73.1\%) received appropriate empirical therapy and 18 (26.9\%) inappropriate therapy. In the former group, 12 received combination therapy with two active drugs (nine meropenem and three imipenem along with colistin [eight] or an active aminoglycoside [four]) and 37 were given one active drug (nine meropenem, five imipenem, fifteen colistin and eight an aminoglycoside). The mortality rates for the patients infected with VIM-positive carbapenem-susceptible organisms were as follows: $8.3 \%(1 / 12)$ for those who received combination therapy with two active drugs, $27 \%$ (10/37) for those who received therapy with one active drug, $27.8 \%$ (5/18) for those who received inappropriate empirical therapy and $28.6 \%(4 / 14)$ for inappropriate definitive therapy. The results 
indicated that the higher mortality observed in patients infected with VIM-positive carbapenem-susceptible K. pneumoniae strains should be probably attributed to the failure to administer an effective combination of antimicrobial agents whenever the MICs of imipenem and meropenem falsely indicated susceptibility $(\leq 4 \mu \mathrm{g} / \mathrm{ml})$.

Fosfomycin, a forgotten antibiotic that inhibits bacterial cell wall biosynthesis, was discovered almost 40 years ago and possesses promising in vitro activity against carbapenem-resistant $P$. aeruginosa and $K$. pneumoniae [40]. In a prospective study fosfomycin was given at $2-4 \mathrm{~g}$ intravenously every $6 \mathrm{~h}$ for $14 \pm 5.6$ days, in combination with other antibiotics, to 11 adult ICU patients with carbapenemresistant $K$. pneumoniae infections without definition of underlying resistance mechanisms [41]. All patients had promising bacteriological and clinical outcomes, with all-cause hospital mortality of $18.2 \%$. No adverse effects were reported. The limited clinical experience with fosfomycin and its safety profile make the performance of well-organized clinical studies almost obligatory. Based on the fact that fosfomycin monotherapy is prohibited due to the prompt emergence of resistance during therapy, the choice of the appropriate adjunctive antibiotic should be carefully investigated in the near future.

A new aminoglycoside (ACHN-490) appears promising in vitro against MDR $K$. pneumoniae, including all KPC producers. It is now in early phase II trials in patients with urinary tract infections [42]. A new $\beta$-lactamase inhibitor, NXL104, shows at least promising in vitro activity against KPC-producing 
Enterobacteriaceae and some other inhibitors are in the pipeline, which unfortunately seems to be very long [43].

Based on the poor situation of 'Bad bugs, no drugs', can clinicians themselves provide some solution to the urgent worldwide problem of antibiotic resistance? There is no doubt that the strict application of infection control measures, particularly hand hygiene, is the cornerstone of nosocomial infection prevention. However, antibiotic stewardship seems to be even more important, since decreasing antibiotic overconsumption results in decreased resistance rates of Gram-negative microorganisms, both in US and European hospitals [44]. It is also evident that in order to prevent resistance, underdosing and prolonged therapy with antimicrobials should be avoided. The pioneering double-blind study of Chastre et al. in 2003 showed that 8 vs 15 days of antibiotic therapy in ventilator-associated pneumonia did not result in excess mortality or recurrent infections, allowed more antibiotic-free days (13.1 \pm 7.4 vs $8.7 \pm 5.2$ days, $P<0.001)$ and resulted in fewer multiresistant pathogens in recurrences $(42.1 \%$ vs $62 \%, P=0.04)$. However, in the case of non-fermenters, 2-week therapy was considered more efficacious [45]. Searching an appropriate marker to permit discontinuation of therapy in ventilator-associated pneumonia, European investigators recently determined in a high number of patients that a procalcitonin value in blood of $<0.25 \mathrm{ng} / \mathrm{ml}$ safely permits discontinuation of antibiotics [46].

It does not need to be emphasized that appropriate cultures should always be taken, pharmacokinetics/pharmacodynamics should be exploited, de-escalation of empirically administered antibiotics should be included as a quality indicator, 
and the role of the infectious diseases specialist should be reconsidered as a vital resource in the implementation of the above strategies.

Funding: The author received an honorarium for writing this article. The funds for the honorarium were provided by Novartis AG, Switzerland and were handled by the organizing committee of the 4th European Conference on Bloodstream Infections for the publication of this supplement.

Competing interests: No conflicts of interest.

Ethical approval: The clinical studies referred to by the author in references 20 , 22 and 35 were approved by the Ethical-Scientific Committees of University General Hospital “ATTIKON”. 


\section{References}

[1] Giamarellou H, Poulakou G. Multidrug-resistant Gram-negative infections: What are the treatment options? Drugs 2009;69:1879-901.

[2] Boucher HW, Talbot GH, Bradley JS, Edwards JE, Gilbert D, Rice LB, et al. Bad bugs, no drugs: no ESKAPE! An update from the Infectious Diseases Society of America. Clin Infect Dis 2009;48:1-12.

[3] National Nosocomial Infections Surveillance (NNIS) System report data summary from January 1992 through June 2004, issued October 2004. Am J Infect Control 2004;32:470-85.

[4] Rossolini GM, Mantengoli E. Treatment and control of severe infections caused by multiresistant Pseudomonas aeruginosa. Clin Microbiol Infect 2005;11(Suppl 4):17-32.

[5] Souli M, Galani I, Giamarellou H. Emergence of extensively drug-resistant and pandrug-resistant Gram-negative bacilli in Europe. Euro Surveill 2008;13(47):pii=19045.

[6] Li J, Nation RL. Old polymyxins are back: is resistance close? Clin Infect Dis 2006;43:663-4.

[7] Kwa A, Kasiakou SK, Tam VH, Falagas ME. Polymyxin B: similarities to and differences from colistin (polymyxin E). Expert Rev Anti Infect Ther 2007;5:81121. 
[8] Gales AC, Jones RN, Sader HS. Global assessment of the antimicrobial activity of polymyxin against 54731 clinical isolates of Gram-negative bacilli: report from the SENTRY Antimicrobial Surveillance Program (2001-4). Clin Microbiol Infect 2006;12:315-21.

[9] Giamarellos-Bourboulis EJ, Sambatakou H, Galani I, Giamarellou H. In vitro interaction of colistin and rifampin on multidrug-resistant Pseudomonas aeruginosa. J Chemother 2003;15:235-8.

[10] Tripodi MF, Durante-Mangoni E, Fortunato R, Utili R, Zarrilli R. Comparative activities of colistin, rifampicin, imipenem and sulbactam/ampicillin alone or in combination against epidemic multidrug-resistant Acinetobacter baumannii isolates producing OXA-58 carbapenemases. Int J Antimicrob Agents 2007;30:537-40.

[11] Tan TY, Ng LS, Tan E, Huang G. In vitro effect of minocycline and colistin combinations on imipenem-resistant Acinetobacter baumannii clinical isolates. $\mathrm{J}$ Antimicrob Chemother 2007;60:421-3.

[12] Souli M, Rekatsina PD, Chryssouli Z, Galani I, Giamarellou H, Kanellakopoulou K. Does the activity of the combination of imipenem and colistin in vitro exceed the problem of resistance in metallo- $\beta$-lactamase-producing Klebsiella pneumoniae isolates? Antimicrob Agents Chemother 2009;53:213335. 
[13] Giamarellou H. Treatment options for multidrug-resistant bacteria. Expert Rev Anti Infect Ther 2006;4:601-18.

[14] Rios FG, Luna CM, Maskin B, Saenz Valiente A, Lloria M, Gando S, et al. Ventilator-associated pneumonia due to colistin susceptible-only microorganisms. Eur Respir J 2007;30:307-13.

[15] Kallel H, Hergafi L, Bahloul M, Hakim A, Dammak H, Chelly H, et al. Safety and efficacy of colistin compared with imipenem in the treatment of ventilatorassociated pneumonia: a matched case-control study. Intensive Care Med 2007;33:1162-7.

[16] Hachem RY, Chemaly RF, Ahmar CA, Jiang Y, Boktour MR, Rjaili GA, et al. Colistin is effective in treatment of infections caused by multidrug-resistant Pseudomonas aeruginosa in cancer patients. Antimicrob Agents Chemother 2007;51:1905-11.

[17] Falagas ME, Rafailidis PI, loannidou E, Alexiou VG, Matthaiou DK, Karageorgopoulos DE, et al. Colistin therapy for microbiologically documented multidrug-resistant Gram-negative bacterial infections: a retrospective cohort study of 258 patients. Int J Antimicrob Agents 2010;35:194-9.

[18] Michalopoulos A, Papadakis E. Inhaled anti-infective agents: emphasis on colistin. Infection 2010;38:81-8.

[19] Cascio A, Conti A, Sinardi L, laria C, Angileri FF, Stassi G, et al. Postneurosurgical multidrug-resistant Acinetobacter baumannii meningitis 
successfully treated with intrathecal colistin: A new case and a systematic review of the literature. Int J Infect Dis 2010;14:e572-9.

[20] Plachouras D, Karvanen M, Friberg LE, Papadomichelakis E, Antoniadou A, Tsangaris I, et al. Population pharmacokinetic analysis of colistin methanesulfonate and colistin after intravenous administration in critically ill patients with infections caused by Gram-negative bacteria. Antimicrob Agents Chemother 2009;53:3430-6.

[21] Alberto C, Mendes C, Burdmann EA. Polymyxins - a review focusing on their nephrotoxicity. Rev Assoc Med Bras 2010;56:752-8.

[22] Antoniadou A, Kontopidou F, Poulakou G, Koratzanis E, Galani I, Papadomichelakis E, et al. Colistin-resistant isolates Klebsiella pneumoniae emerging in intensive care unit patients: first report of a multiclonal cluster. $\mathrm{J}$ Antimicrob Chemother 2007;59:786-90.

[23] Giamarellou H. Colistin: the loss of the last frontier? APUA Newsletter 2007;25:5.

[24] Souli M, Kontopidou FV, Papadomichelakis E, Galani I, Armaganidis A, Giamarellou H. Clinical experience of serious infections caused by Enterobacteriaceae producing VIM-1 metallo-beta-lactamase in a Greek university hospital. Clin Infect Dis 2008;46:847-54.

[25] Souli M, Galani I, Antoniadou A, Papadomichelakis E, Poulakou G, Panagea $\mathrm{T}$, et al. An outbreak of infection due to beta-lactamase Klebsiella pneumoniae 
carbapenemase 2-producing K. pneumoniae in a Greek university hospital: molecular characterization, epidemiology, and outcomes. Clin Infect Dis 2010;50:364-73.

[26] Mentzelopoulos SD, Pratikaki M, Platsouka E, Kraniotaki H, Zervakis D, Koutsoukou A, et al. Prolonged use of carbapenems and colistin predisposes to ventilator-associated pneumonia by pandrug-resistant Pseudomonas aeruginosa. Intensive Care Med 2007;33:1524-32.

[27] Matthaiou DK, Michalopoulos A, Rafailidis PI, Karageorgopoulos DE, Papaioannou V, Ntani G, et al. Risk factors associated with the isolation of colistin-resistant Gram-negative bacteria: a matched case-control study. Crit Care Med 2008;36:807-11.

[28] Souli M, Kontopidou FV, Koratzanis E, Antoniadou A, Giannitsioti E, Evangelopoulou $\mathrm{P}$, et al. In vitro activity of tigecycline against multiple-drugresistant, including pan-resistant, Gram-negative and Gram-positive clinical isolates from Greek hospitals. Antimicrob Agents Chemother 2006;50:3166-9.

[29] Milatovic D, Schmitz FJ, Verhoef J, Fluit AC. Activities of the glycylcycline tigecycline (GAR-936) against 1,924 recent European clinical bacterial isolates. Antimicrob Agents Chemother 2003;47:400-4.

[30] Bouchillon SK, Iredell JR, Barkham T, Lee K, Dowzicky MJ. Comparative in vitro activity of tigecycline and other antimicrobials against Gram-negative and Gram-positive organisms collected from the Asia-Pacific Rim as part of the 
Tigecycline Evaluation and Surveillance Trial (TEST). Int J Antimicrob Agents 2009;33:130-6.

[31] Liu JW, Wang LS, Cheng YJ, Hsu GJ, Lu PL, Liu YC, et al. In-vitro activity of tigecycline against clinical isolates of Acinetobacter baumannii in Taiwan. Int $\mathrm{J}$ Antimicrob Agents 2008;32(Suppl 3):S188-91.

[32] Castanheira M, Sader HS, Deshpande LM, Fritsche TR, Jones RN.

Antimicrobial activities of tigecycline and other broad-spectrum antimicrobials tested against serine carbapenemase- and metallo-beta-lactamase-producing Enterobacteriaceae: report from the SENTRY Antimicrobial Surveillance Program. Antimicrob Agents Chemother 2008;52:570-3.

[33] Meagher AK, Ambrose PG, Grasela TH, Ellis-Grosse EJ.

Pharmacokinetic/pharmacodynamic profile for tigecycline_a new glycylcycline antimicrobial agent. Diagn Microbiol Infect Dis 2005;52:165-71.

[34] MacGowan AP. Tigecycline pharmacokinetic/pharmacodynamic update. J Antimicrob Chemother 2008;62(Suppl 1):i11-6.

[35] Poulakou G, Kontopidou FV, Paramythiotou E, Kompoti M, Katsiari M, Mainas E, et al. Tigecycline in the treatment of infections from multi-drug resistant Gram-negative pathogens. J Infect 2009;58:273-84.

[36] Ellis-Grosse EJ, Babinchak T, Dartois N, Rose G, Loh E, Tigecycline 300 cSSSI Study Group, et al. The efficacy and safety of tigecycline in the treatment of skin and skin-structure infections: results of 2 double-blind phase 3 
comparison studies with vancomycin-aztreonam. Clin Infect Dis 2005;41(Suppl 5):S341-53.

[37] Peleg AY, Potoski BA, Rea R, Adams J, Sethi J, Capitano B, et al. Acinetobacter baumannii bloodstream infection while receiving tigecycline: a cautionary report. J Antimicrob Chemother 2007;59:128-31.

[38] Carmeli Y, Akova M, Cornaglia G, Daikos GL, Garau J, Harbarth S, et al. Controlling the spread of carbapenemase-producing Gram-negatives: therapeutic approach and infection control. Clin Microbiol Infect 2010;16:102-11.

[39] Daikos GL, Petrikkos P, Psichogiou M, Kosmidis C, Vryonis E, Skoutelis A, et al. Prospective observational study of the impact of VIM-1 metallo- $\beta$-lactmase on the outcome of patients with Klebsiella pneumoniae bloodstream infections. Antimicrob Agents Chemother 2009;53:1868-73.

[40] Falagas ME, Maraki S, Karageorgopoulos DE, Kastoris AC, Mavromanolakis E, Samonis G. Antimicrobial susceptibility of multidrug-resistant (MDR) and extensively drug-resistant (XDR) Enterobacteriaceae isolates to fosfomycin. Int $\mathrm{J}$ Antimicrob Agents 2010;35:240-3.

[41] Michalopoulos A, Virtzili S, Rafailidis P, Chalevelakis G, Damala M, Falagas ME. Intravenous fosfomycin for the treatment of nosocomial infections caused by carbapenem-resistant Klebsiella pneumoniae in critically ill patients: a prospective evaluation. Clin Microbiol Infect 2010;16:184-6. 
[42] Endimiani A, Hujer KM, Hujer AM, Armstrong ES, Choudhary Y, Aggen JB, et al. ACHN-490, a neoglycoside with potent in vitro activity against multidrugresistant Klebsiella pneumoniae isolates. Antimicrob Agents Chemother 2009;53:4504-7.

[43] Stachyra T, Levasseur P, Péchereau MC, Girard AM, Claudon M, Miossec C, et al. In vitro activity of the $\beta$-lactamase inhibitor NXL104 against KPC-2 carbapenemase and Enterobacteriaceae expressing KPC carbapenemases. J Antimicrob Chemother 2009;64:326-9.

[44] Pakyz AL, Oinonen M, Polk RE. Relationship of carbapenem restriction in 22 university teaching hospitals to carbapenem use and carbapenem-resistant Pseudomonas aeruginosa. Antimicrob Agents Chemother 2009;53:1983-6.

[45] Chastre J, Wolff M, Fagon JY, Chevret S, Thomas F, Wermert D, et al. Comparison of 8 vs 15 days of antibiotic therapy for ventilator-associated pneumonia in adults: a randomized trial. JAMA 2003;290:2588-98.

[46] Bouadma L, Luyt CE, Tubach F, Cracco C, Alvarez A, Schwebel C, et al. Use of procalcitonin to reduce patients' exposure to antibiotics in intensive care units (PRORATA trial): a multicentre randomised controlled trial. Lancet 2010;375:463-74. 